\title{
Existence theorems for second-order radial epiderivatives
}

\author{
Gonca Inceoglu \\ Department of Mathematics and Science Education, Anadolu University, Turkey
}

Received: 24 February 2017, Accepted: 24 April 2017

Published online: 2 May 2017.

\begin{abstract}
In this paper, we introduce the concepts of second-order radial epiderivative and second-order generalized radial epiderivative for nonconvex set-valued maps. We also investigate some of their properties. We give existence theorems for the second-order generalized radial epiderivatives.
\end{abstract}

Keywords: Second-order radial set, second-order generalized radial epiderivative, second-order radial epiderivative.

\section{Introduction}

In set-valued analysis the notion of derivative for set-valued maps has been formulated in different ways ( see [1] to [31]). Kasimbeyli introduced in [22] the notion of the radial epiderivative of a set-valued map, with its help formulated optimality conditins in single valued and set-valued optimization without convexity assumption. This definition of the radial epiderivative given by Kasımbeyli is different from that of Flores- Bazan [7] and is similar to the definition of the contingent epiderivative given by Jahn and Rauh [19].

Kasımbeyli and İnceoğlu introduced in [24] the notion of generalized radial epiderivative for set-valued maps and investigated existence conditions for generalized radial epiderivative. They established the relationship between the radial epiderivative and the generalized radial epiderivative. By using the generalized radial epiderivative, Kasımbeyli and İnceoğlu presented the necessary and sufficient optimality conditions for set-valued optimization.

In the last years, the second-order optimality condition and higher-order optimality conditions have been widely investigated in set-valued optimization [3], [4], [5], [6],[9],[11],[12], [13], [14], [16],[18],[25], [23], [21], [28], [31]. It can be seen that a second-order contingent set, introduced by Aubin and Frankowska [2], and a second-order asymptotic contingent cone, introduced by Penot [28], play a important role in establishing second-order optimality conditions. Jahn et al. proposed the second-order contingent derivative and the second-order contingent epiderivative in terms of the second-order contingent set [18], introduced by Aubin and Frankowska [2]. They obtained the second-order optimality conditions by using these derivatives in set-valued optimization.

Khan and Tammer gave new second-order optimality conditions in set-valued optimization. They presented an extension of the well-known Dubovitski-Milutin approach to set-valued optimization [25].

Anh and Khanh introduced the higher-order radial sets and corresponding derivatives. They proposed their properties and basic calculus rules. They established both necessary and sufficient higher-order conditions for weak efficiency in 
set-valued vector optimization problem [4].

Anh and Khanh gave both necessary and sufficient higher-order conditions for various kinds of proper solutions to nonsmooth vector optimization problem in terms of higher-order radial sets and radial derivatives [5]. However, the results of second-order radial epiderivatives for set-valued optimization problems still need to be addressed.

Motivated by the work above in [4], [5], [18], [25], we introduce the notions of second-order radial epiderivative and the second-order generalized radial epiderivative and discuss the relationship the second-order radial epiderivative and the second-order generalized radial epiderivative. We also have existence theorems of second-order radial epiderivatives. This paper is divided into three sections. In Section 2, we recall some basic concepts. In Section 3, we introduce the second-order radial epiderivative and the second-order generalized radial epiderivative and give the existence theorems and some of their basic properties.

\section{Preliminaries}

Throughout this paper, let $\left(X,\|\cdot\|_{X}\right)$ and $\left(Y,\|\cdot\|_{Y}\right)$ be real normed spaces and let $Y$ be partially ordered by a closed convex pointed cone $C \subset Y$. Let $F: X \rightarrow 2^{Y}$ be a set-valued map, let $(\bar{x}, \bar{y}) \in \operatorname{graph}(F)$, let $(\bar{u}, \bar{v}) \in X \times Y$.

We recall the concept of the radial epiderivative and the generalized radial epiderivative introduced by Kasımbeyli [22], and Kasımbeyli and İnceoğlu [24], respectively, together with some standard notions.

Definition 1. Let $U$ be a nonempty subset of a real normed space $\left(Z,\|\cdot\|_{Z}\right)$, and let $\bar{z} \in \operatorname{cl}(U)$ (closure of $U$ ) be a given element. The closed radial cone $R(U, \bar{z})$ of $U$ at $\bar{z} \in c l(U)$ is the set of all $z \in Z$ such that there are $\lambda_{n}>0$ and a sequence $\left(z_{n}\right)_{n \in \mathbb{N}} \subset Z$ with $\lim _{n \rightarrow \infty} z_{n}=z$ so that $\bar{z}+\lambda_{n} z_{n} \in U$, for all $n \in \mathbb{N}$ [7], [24], [30].

It follows from this definitions that $R(U, \bar{z})=\operatorname{cl}($ cone $(U-\bar{z}))$, where cone denotes the conic hull of a set, which is the smallest cone containing $U-\bar{z}$ [7], [8], [24].

Definition 2. Let $\left(X,\|\cdot\|_{X}\right)$ and $\left(Y,\|\cdot\|_{Y}\right)$ be real normed spaces, let $F: X \rightarrow 2^{Y}$ be a set-valued map.

(i) The set

$$
\operatorname{graph}(F)=\{(x, y) \in X \times Y \mid y \in F(x)\}
$$

is called the graph of $F$;

(ii) The set

$$
\operatorname{dom}(F)=\{x \in X \mid F(x) \neq \emptyset\}
$$

is called the domain of $F$;

(iii) Let $Y$ be partially ordered by a proper, convex, and pointed cone $C \subset Y$. The set

$$
\operatorname{epi}(F)=\{(x, y) \in X \times Y \mid y \in F(x)+C\}
$$

is called the epigraph of $F$,

(iv) Let $C \subset Y$ a proper, convex and pointed cone. The profile map $P_{F}: X \rightarrow 2^{Y}$ is defined by

$$
P_{F}(x)=F(x)+C
$$

for every $x \in \operatorname{dom}(F)$.

(v) Let $(\bar{x}, \bar{y}) \in \operatorname{graph}(F)$. A set valued map $D_{R} F(\bar{x}, \bar{y}): X \rightarrow 2^{Y}$ whose graph coincides with the contingent cone to graph of $F$ at $(\bar{x}, \bar{y})$, that is

$$
\operatorname{graph}\left(D_{R} F(\bar{x}, \bar{y})\right)=R(\operatorname{graph}(F),(\bar{x}, \bar{y})),
$$


is called radial derivative of $F$ at $(\bar{x}, \bar{y})[7],[30]$.

Now, we give the definition of the radial epiderivative given by Kasımbeyli without convexity and boundedness [22].

Definition 3. Let $Y$ be partially ordered by a convex cone $C \subset Y$, let $S$ be a nonempty subset of $X$ and let $F: S \rightarrow 2^{Y}$ be a set-valued map. Let a pair $(\bar{x}, \bar{y}) \in$ graph $(F)$ be given. A single-valued map $D_{r} F(\bar{x}, \bar{y}): X \rightarrow Y$ whose epigraph equals the radial cone to the epigraph of $F$ at $(\bar{x}, \bar{y})$, i.e.

$$
\operatorname{epi}\left(D_{r} F(\bar{x}, \bar{y})\right)=R(\operatorname{epi}(F),(\bar{x}, \bar{y}))
$$

is called radial epiderivative of $F$ at $(\bar{x}, \bar{y})$.

To give the definition of the generalized radial epiderivative, we recall the minimality concept [17],[26].

Definition 4. Let $\left(Y,\|\cdot\|_{Y}\right)$ be a real normed space partially ordered by a convex cone $C \subset Y$. Let $D$ be a subset of $Y$ and let $\bar{y} \in D$.

(i) The element $\bar{y}$ is said to be a minimal element of $D$, if $D \cap(\{\bar{y}\}-C)=\{\bar{y}\}$.

(ii) Let the ordering cone have a nonempty interior int $(C)$. The element $\bar{y}$ is said to be a weakly minimal element of $D$, if $D \cap(\{\bar{y}\}-$ int $(C))=\emptyset$. The set of all minimal, weakly minimal elements of $D$ with respect to the ordering cone $C$ is denoted by $\operatorname{Min} D, W-M i n D$, respectively.

Now, we recall the generalized radial epiderivative for set-valued maps given by Kasımbeyli and İnceoğlu in [24].

Definition 5. A set valued map $D_{g r} F(\bar{x}, \bar{y}): X \rightarrow 2^{Y}$ is called the generalized radial epiderivative of $F$ at $(\bar{x}, \bar{y})$ if

$$
D_{g r} F(\bar{x}, \bar{y})(x)=\operatorname{Min}(G(x), C),
$$

where $G: X \rightarrow 2^{Y}$ is the set-valued map given by

$$
G(x)=\{y \in Y \mid(x, y) \in R(e p i(F),(\bar{x}, \bar{y}))\}, \forall x \in X .
$$

\section{Second-order radial set and second-order radial epiderivatives}

In this section, we propose the definitions of the second-order radial epiderivatives. By using these definitions, we prove existence theorem and give some of their properties and optimality conditions.

Anh and Khanh defined $m$-th-order radial set and $m$-th-order radial derivative [5]. Based on this, we give the following definitions of second-order radial set and second-order radial derivative.

Definition 6. Let $\left(X,\|\cdot\|_{X}\right)$ be a real normed space, let $S$ be a nonempty subset of $X$, let $\bar{x} \in \operatorname{cl}(S)$ and let $w \in X$ The second-order radial set of $S$ at $\bar{x}$ with respect to $w$ is

$$
R^{2}(S, \bar{x}, w)=\left\{x \in X \mid \exists t_{n}>0, \exists x_{n} \rightarrow x, \forall n, \bar{x}+t_{n} w+t_{n}^{2} x_{n} \in S\right\} .
$$

It is also clear that $R^{2}\left(S, \bar{x}, 0_{X}\right)=R(S, \bar{x}), 0_{X}$ the zero element of $X$.

The following definition was presented by Ha in [15]. 
Definition 7. Let $F: X \rightarrow 2^{Y}$ be a set-valued map, let $(\bar{x}, \bar{y}) \in \operatorname{graph}(F)$, let $(\bar{u}, \bar{v}) \in X \times Y$. The second-order radial derivative of $F$ at $(\bar{x}, \bar{y})$ with respect to $(\bar{u}, \bar{v})$ is the set-valued map $D_{R}^{2} F(\bar{x}, \bar{y}, \bar{u}, \bar{v}): X \rightarrow 2^{Y}$ whose graph is

$$
\operatorname{graph}\left(D_{R}^{2} F(\bar{x}, \bar{y}, \bar{u}, \bar{v})\right)=R^{2}(\operatorname{graph}(F),(\bar{x}, \bar{y}),(\bar{u}, \bar{v}))
$$

The relation (2) can be expressed equivalently by

$$
D_{R}^{2} F(\bar{x}, \bar{y}, \bar{u}, \bar{v})(x)=\left\{\begin{array}{c}
y \in Y \mid \exists t_{n}>0, \exists x_{n} \rightarrow x, \exists y_{n} \rightarrow y, \forall n \\
\bar{y}+t_{n} \bar{v}+t_{n}^{2} y_{n} \in F\left(\bar{x}+t_{n} \bar{u}+t_{n}^{2} x_{n}\right)
\end{array}\right\}
$$

The following definition is a generalization given by Kasımbeyli and Kasımbeyli and İnceoğlu, respectively [22],[?].

Definition 8. Let $F: X \rightarrow 2^{Y}$ be a set-valued map, let $(\bar{x}, \bar{y}) \in \operatorname{graph}(F)$, let $(\bar{u}, \bar{v}) \in X \times Y$.

(i) A single-valued map $D_{r}^{2} F(\bar{x}, \bar{y}, \bar{u}, \bar{v}): X \rightarrow Y$ whose epigraph equals the second-order radial set to the epigraph of $F$ at $(\bar{x}, \bar{y})$ with respect to $(\bar{u}, \bar{v})$, i.e.,

$$
\operatorname{epi}\left(D_{r}^{2} F(\bar{x}, \bar{y}, \bar{u}, \bar{v})\right)=R^{2}(\operatorname{epi}(F),(\bar{x}, \bar{y}),(\bar{u}, \bar{v}))
$$

is called the second-order radial epiderivative.

(ii) A set-valued map $D_{g r}^{2} F(\bar{x}, \bar{y}, \bar{u}, \bar{v}): X \rightarrow 2^{Y}$ is called the second-order generalized radial epiderivative of $F$ at $(\bar{x}, \bar{y})$ with respect to $(\bar{u}, \bar{v})$ if

$$
D_{g r}^{2} F(\bar{x}, \bar{y}, \bar{u}, \bar{v})(x)=\operatorname{Min}\left(G^{2}(x), C\right), x \in \operatorname{dom}\left(G^{2}(x)\right),
$$

where $G^{2}: X \rightarrow 2^{Y}$ is a set-valued map defined by

$$
G^{2}(x)=\left\{y \in Y \mid(x, y) \in R^{2}(e p i(F),(\bar{x}, \bar{y}),(\bar{u}, \bar{v}))\right\}
$$

Example 1. Let $F: \mathbb{R} \rightarrow 2^{\mathbb{R}}$ be a set-valued map given by

$$
F(x)=\{y \in \mathbb{R} \mid y \geq x\}, \text { for all } x \in \mathbb{R}
$$

Let $(\bar{x}, \bar{y})=(0,0)$ and let $(\bar{u}, \bar{v})=(1,0)$. Then

$$
R^{2}(e p i(F),(0,0),(1,0))=\left\{c z \in \mathbb{R}^{2} \mid \exists t_{n}>0, \exists\left(z_{n}\right) \rightarrow z, \text { for all } n, t_{n}(1,0)+t_{n}^{2} z_{n} \in e p i F\right\} .
$$

The condition

$$
t_{n}(1,0)+t_{n}^{2} z_{n} \in e p i(F)
$$

is equivalent to

$$
t_{n}^{2} z_{n_{2}} \geq t_{n}+t_{n}^{2} z_{n_{1}}
$$

hence,

$$
z_{n_{2}} \geq\left(1+t_{n} z_{n_{1}}\right)^{2}
$$

Since $t_{n}>0$ and $z_{n_{2}} \rightarrow z_{2}, z_{n_{1}} \rightarrow z_{1}$, we obtain that

$$
R^{2}(e p i(F),(0,0),(1,0))=\mathbb{R} \times[1,0) .
$$

Consequently, we have

$$
G^{2}(x)=[1,0),
$$


for every $x \in \mathbb{R}$. On the other hand,

$$
D_{r}^{2} F(0,0,1,0)(x)=\{1\},
$$

for every $x \in \mathbb{R}$.

$$
D_{g r}^{2} F(0,0,1,0)(x)=\operatorname{Min}\left(G^{2}(x), \mathbb{R}_{+}\right)=\{1\}
$$

for every $x \in \mathbb{R}$.

Proposition 1. For every $x \in \operatorname{dom}\left(D_{R}^{2} F(\bar{x}, \bar{y}, \bar{u}, \bar{v})\right)$, the following inclusion holds:

$$
D_{R}^{2} F(\bar{x}, \bar{y}, \bar{u}, \bar{v})(x)+C_{Y} \subseteq D_{R}^{2} P_{F}(\bar{x}, \bar{y}, \bar{u}, \bar{v})(x) .
$$

Proof. Let $y \in D_{R}^{2} F(\bar{x}, \bar{y}, \bar{u}, \bar{v})(x)$ and let $c \in C$ be arbitrarily chosen. Then, we have

$$
(x, y) \in R^{2}(\operatorname{graph}(F),(\bar{x}, \bar{y}),(\bar{u}, \bar{v})) .
$$

This means the existence of $t_{n}>0$ and $\left(x_{n}, y_{n}\right) \rightarrow(x, y)$ such that for all $n \in \mathbb{N}$

$$
\bar{y}+t_{n} \bar{v}+t_{n}^{2} y_{n} \in F\left(\bar{x}+t_{n} \bar{u}+t_{n}^{2} x_{n}\right) .
$$

Setting

$$
\bar{y}_{n}=y_{n}+c,
$$

we get that

$$
\bar{y}+t_{n} \bar{v}+t_{n}^{2} \bar{y}_{n}=\bar{y}+t_{n} \bar{v}+t_{n}^{2} y_{n}+t_{n}^{2} c \in F\left(\bar{x}+t_{n} \bar{u}+t_{n}^{2} x_{n}\right)+C,
$$

and this ensures that

$$
(\bar{x}, \bar{y})+t_{n}(\bar{u}, \bar{v})+t_{n}^{2}\left(x_{n}, y_{n}\right) \in \operatorname{epi}(F) .
$$

As $\bar{y}_{n} \rightarrow y+c$, we conclude that

$$
y+c \in D_{R}^{2} P_{F}(\bar{x}, \bar{y}, \bar{u}, \bar{v})(x) .
$$

This completes the proof.

Corollary 1. For every $x \in \operatorname{dom}\left(D_{R}^{2} P_{F}(\bar{x}, \bar{y}, \bar{u}, \bar{v})\right)$, the following inclusion holds:

$$
D_{R}^{2} P_{F}(\bar{x}, \bar{y}, \bar{u}, \bar{v})(x)+C_{Y}=D_{R}^{2} P_{F}(\bar{x}, \bar{y}, \bar{u}, \bar{v})(x)
$$

Proof.The inclusion

$$
D_{R}^{2} P_{F}(\bar{x}, \bar{y}, \bar{u}, \bar{v})(x) \subseteq D_{R}^{2} P_{F}(\bar{x}, \bar{y}, \bar{u}, \bar{v})(x)+C
$$

is trivial. From Proposition 1 and the identity $P_{F}()+C=.P_{F}($.$) , we get this inclusion$

$$
D_{R}^{2} P_{F}(\bar{x}, \bar{y}, \bar{u}, \bar{v})(x)+C \subseteq D_{R}^{2} P_{F}(\bar{x}, \bar{y}, \bar{u}, \bar{v})(x) .
$$

Before we give an existence theorems for second-order generalized radial epiderivative, we remind that a convex cone $C$ is called regular (see ref. [27]) if each $C$-decreasing and $C$-lower bounded sequence converges to an element of $C$.

The proof of the next theorem will be used the following lemma given by Sonntag-Zalinescu in [29].

Lemma 1. Let $Y$ be a real normed space and let $D \subset Y$. Then 
(i) If the cone $C$ is regular and $D$ is closed and $C$-lower bounded, then $\min (D, C) \neq \emptyset$ and the domination property holds, that is, $D \subset \min (D, C)+C$.

(ii) Let the cone $C$ has a compact base and let $D$ be closed and convex. If $\min (D, C) \neq \emptyset$ then the domination property holds.

Next, we give the existence theorems for second-order generalized radial epiderivative

Theorem 1. Let the convex cone $C \subset Y$ be regular. For every $x \in \operatorname{dom}\left(G^{2}\right)$, let the set $D_{g r}^{2} F(\bar{x}, \bar{y}, \bar{u}, \bar{v})(x)$ have a $C$-lower bound. Then for every $x \in \operatorname{dom}\left(G^{2}\right), D_{g r}^{2} F(\bar{x}, \bar{y}, \bar{u}, \bar{v})(x)$ exists. Moreover, the following equality holds:

$$
\operatorname{epi}\left(D_{g r}^{2} F(\bar{x}, \bar{y}, \bar{u}, \bar{v})\right)=R^{2}(\operatorname{epi}(F),(\bar{x}, \bar{y}),(\bar{u}, \bar{v})) .
$$

Proof. Because the second-order radial set is always closed in a real normed space, the set $G^{2}(x)$ is closed. As $G^{2}(x)$ has a $C$ - lower bound and $C$ is a regular cone, we have $D_{g r}^{2} F(\bar{x}, \bar{y}, \bar{u}, \bar{v})(x) \neq \emptyset$ by the Lemma 1 . Moreover, by the same lemma,

$$
G^{2}(x) \subseteq D_{g r}^{2} F(\bar{x}, \bar{y}, \bar{u}, \bar{v})(x)+C,
$$

for every $x \in \operatorname{dom}\left(G^{2}(x)\right)$.

For the inverse inclusion, let $y \in D_{g r}^{2} F(\bar{x}, \bar{y}, \bar{u}, \bar{v})(x)+C$, which implies that

$$
y \in G^{2}(x)+C
$$

It follows from the definition of the second-order radial derivative that

$$
G^{2}(x)=D_{R}^{2} P_{F}(\bar{x}, \bar{y}, \bar{u}, \bar{v})(x)
$$

From (8) and (9), we get

$$
y \in D_{R}^{2} P_{F}(\bar{x}, \bar{y}, \bar{u}, \bar{v})(x)+C .
$$

In view of Corollary $1 y \in D_{R}^{2} P_{F}(\bar{x}, \bar{y}, \bar{u}, \bar{v})(x)=G^{2}(x)$, which confirms the inclusion

$$
D_{g r}^{2} F(\bar{x}, \bar{y}, \bar{u}, \bar{v})(x)+C \subseteq D_{R}^{2} P_{F}(\bar{x}, \bar{y}, \bar{u}, \bar{v})(x) .
$$

Therefore,

$$
D_{g r}^{2} F(\bar{x}, \bar{y}, \bar{u}, \bar{v})(x)+C=G^{2}(x)=D_{R}^{2} P_{F}(\bar{x}, \bar{y}, \bar{u}, \bar{v})(x) .
$$

From (11) and the definition of the second-order radial derivative,

$$
\operatorname{epi}\left(D_{g r}^{2} F(\bar{x}, \bar{y}, \bar{u}, \bar{v})\right)=\operatorname{graph}\left(D_{R}^{2} P_{F}(\bar{x}, \bar{y}, \bar{u}, \bar{v})(x)\right)=R^{2}\left(\operatorname{graph}\left(P_{F}\right),(\bar{x}, \bar{y}),(\bar{u}, \bar{v})\right)=R^{2}(\operatorname{epi}(F),(\bar{x}, \bar{y}),(\bar{u}, \bar{v})),
$$

which complete the proof.

Proposition 2. Let the convex cone $C \subset Y$ be regular. Let $F: X \rightarrow 2^{Y}$ be a set-valued map, let $(\bar{x}, \bar{y}) \in$ graph $(F)$, let $(\bar{u}, \bar{v}) \in X \times Y$. For every $x \in \operatorname{dom}\left(G^{2}(x)\right)$, let the set $G^{2}(x)$ have a $C$-lower bound. The following assertion is satisfied:

$$
\operatorname{epi}\left(D_{g r}^{2} F(\bar{x}, \bar{y}, \bar{u}, \bar{v})\right) \subset R^{2}(\operatorname{dom}(F), \bar{x}, \bar{u}) \times Y .
$$


Proof. Let $(\bar{x}, \bar{y}) \in \operatorname{epi}\left(D_{g r}^{2}(\bar{x}, \bar{y}, \bar{u}, \bar{v})\right)$. Then $(\bar{x}, \bar{y}) \in R^{2}($ epi $(f),(\bar{x}, \bar{y}),(\bar{u}, \bar{v}))$ It follows from the definition of the secondorder generalized radial epiderivative that there exist sequences $t_{n}>0$ and $\left(x_{n}, y_{n}\right)$ with $\left(x_{n}, y_{n}\right) \rightarrow(x, y)$ such that

$$
\begin{gathered}
(\bar{x}, \bar{y})+t_{n}(\bar{u}, \bar{v})+t_{n}^{2}\left(x_{n}, y_{n}\right) \in \text { epi }(F), \text { forall } n \in N, \\
\bar{y}+t_{n} \bar{v}+t_{n}^{2} \in F\left(\bar{x}+t_{n} \bar{u}+t_{n}^{2} x_{n}\right)+C, \text { forall } n \in N .
\end{gathered}
$$

Therefore we have $\bar{x}+t_{n} \bar{u}+t_{n}^{2} x_{n} \in \operatorname{dom}(F)$. This implies that $(x, y) \in R^{2} \operatorname{dom}(F), \bar{x}, \bar{u} \times Y$.

Proposition 3. Let $A \subset X$ be nonempty set and let $C \subset Y$ be a convex cone with int $(C) \neq \emptyset$. Let $F: A \subset X \rightarrow 2^{Y}$ be a set-valued map, let $E=\operatorname{dom}\left(D_{g r}^{2} F(\bar{x}, \bar{y}, \bar{u}, \bar{v})\right)$. Then

$$
\bigcup_{x \in E} D_{g r}^{2} F(\bar{x}, \bar{y}, \bar{u}, \bar{v}) \subset R^{2}(F(A)+C, \bar{y}, \bar{v})
$$

Proof. Let $y \in D_{g r}^{2} F(\bar{x}, \bar{y}, \bar{u}, \bar{v})(E)$ and let $x \in E$ be the corresponding element such that $y \in D_{g r}^{2} F(\bar{x}, \bar{y}, \bar{u}, \bar{v})(x)$. Then, $(x, y) \in R^{2}(\operatorname{epi}(F),(\bar{x}, \bar{y}),(\bar{u}, \bar{v}))$. There exist $t_{n}>0,\left(x_{n}, y_{n}\right) \rightarrow(x, y)$ such that ,for all $n \in N$,

$$
\bar{y}+t_{n} \bar{v}+t_{n}^{2} y_{n} \in F\left(\bar{x}+t_{n} \bar{u}+t_{n}^{2} x_{n}\right)+C \subset F(A)+C
$$

Since $\lambda_{n}>0$ and $y_{n} \rightarrow y$, we get $y \in R^{2}(F(A)+C, \bar{y}, \bar{v})$. Because $y$ is chosen arbitrarily, we have $D_{g r}^{2} F(\bar{x}, \bar{y}, \bar{u}, \bar{v})(E) \subset$ $R^{2}(F(A)+C, \bar{y}, \bar{v})$.

The following result establish relationship between the second-order radial epiderivative and the second-order generalized radial epiderivative.

Proposition 4. Assume that the second-order radial epiderivative $D_{r}^{2} F(\bar{x}, \bar{y}, \bar{u}, \bar{v})$ of $F: X \rightarrow 2^{Y}$ at $(\bar{x}, \bar{y}) \in$ graph $(F)$ with respect to $(\bar{u}, \bar{v}) \in X \times Y$ exist. Then

$$
D_{g r}^{2} F(\bar{x}, \bar{y}, \bar{u}, \bar{v})(x)=\operatorname{Min}\left(D_{r}^{2} F(\bar{x}, \bar{y}, \bar{u}, \bar{v}), C_{Y}\right)
$$

for all $x \in \operatorname{dom}\left(D_{r}^{2} F(\bar{x}, \bar{y}, \bar{u}, \bar{v})\right)$.

Proof. It follows from the Definition 8 that $D_{r}^{2} F(\bar{x}, \bar{y}, \bar{u}, \bar{v})$

$$
\operatorname{epi}\left(D_{r}^{2} F(\bar{x}, \bar{y}, \bar{u}, \bar{v})\right)=R^{2}(\operatorname{epi}(F),(\bar{x}, \bar{y}),(\bar{u}, \bar{v}))=\operatorname{graph}\left(D_{R}^{2} P_{F}(\bar{x}, \bar{y}, \bar{u}, \bar{v})\right) .
$$

Hence,

$$
\left\{D_{r}^{2} F(\bar{x}, \bar{y}, \bar{u}, \bar{v})(x)\right\}+C_{Y}=D_{R}^{2} P_{F}(\bar{x}, \bar{y}, \bar{u}, \bar{v})(x),
$$

for every $x \in \operatorname{dom}\left(D_{R}^{2} P_{F}(\bar{x}, \bar{y}, \bar{u}, \bar{v})\right)$. In view of the Definition 8 and the (12) equality, the second-order generalized radial epiderivative $D_{g r}^{2} F(\bar{x}, \bar{y}, \bar{u}, \bar{v}): X \rightarrow 2^{Y}$ is given by

$$
D_{g r}^{2} F(\bar{x}, \bar{y}, \bar{u}, \bar{v})(x)=\operatorname{Min}\left(D_{r}^{2} F(\bar{x}, \bar{y}, \bar{u}, \bar{v}), C_{Y}\right) .
$$

\section{Conclusion}

In this study, we introduced the two new concepts called second-order radial epiderivative and second-order generalized radial epiderivative. We gave some properties of these epiderivatives. We proved the existence theorems for second-order radial epiderivatives. 


\section{Competing interests}

The authors declare that they have no competing interests.

\section{Authors' contributions}

All authors have contributed to all parts of the article. All authors read and approved the final manuscript.

\section{References}

[1] Aubin, J.P.,1981, Contingent Derivatives of Set-Valued Maps and Existence of Solutions to Nonlinear Inclusions and Differential Inclusions. In: Nachbin, L (ed.) Mathematics Analysis and Applications, part A, 160-229, Academic Press, New York.

[2] Aubin, J.P., Frankowska, H., 1990, Set Valued Analysis,Birkhauser, Boston.

[3] Aghezzaf, B. and Hachimi, M., 1999, Second Order Optimality Conditions in Multiobjective Optimization Problems,J. Optim. Theory Apply., 102,1,37-50.

[4] Anh, N.L.H., and Khanh, P.Q., 2013, Higher-Order Optimality Conditions in Set-Valued optimization Using Radial Sets and Radial Derivatives. J. Glob Optim.,56,2,519-536.

[5] Anh, N.L.H. and Khanh, P.Q., 2014, Higher-Order optimality Conditions for Proper Efficiency in Nonsmooth Vector Optimization Using Radial Sets and Radial Derivatives,J. Glob Optim., 58,4, 693-709.

[6] Anh, N.L.H. Khanh, P.Q. and Tung, L.T., 2011, Higher-Order Radial Derivatives and Optimality Conditions in Nonsmooth Vector Optimization, Nonlinear Anal.Theory Meth.Appl.,74,7365-7379.

[7] Bazan, F.F., 2001, Optimality Conditions in Nonconvex Set-Valued Optimization, Mathematical Methods of Operations Research,53, 403-417.

[8] Bazan, F.F., 2003, Radial Epiderivatives and Asymptotic Functions in Nonconvex Vector Optimization, SIAM J. Optimization, 14,284-305.

[9] Bigi, G. and Castellani, M.,2000, Second Order Optimality Conditions for Differentiable Multiobjective Problems, RARIO Operations Research, 34,411-426.

[10] Chen, G.Y. and Jahn, J., 1998, Optimality Conditions for Set-Valued Optimization Problems, Mathematical Methods of Operations Research, 48,187-200.

[11] Cambini, A. and Martein, L., 2002, First and Second Order Optimality Conditions in Vector Optimization,Journal of Statistics and Management Systems,5,295-319.

[12] Cambini, A., Martein, L. and Vlach,M., 1999, Second Order Tangent Sets and Optimality Conditions, Matematica Japonica, 49,451-461.

[13] Giorgi, G., Jimenez, B. and Novo, V., 2010, An Overview of Second Order Tangent Sets and Their Application to Vector Optimization, SeMA Journal,52, 1, 73-96.

[14] Gutierrez,C., Jimenez,B. and Novo, V., 2009, New Second-Order Directional Derivative and Optimality Conditions in Scalar and Vector Optimization, J. Optim. Theory Appl., 142,85-106.

[15] Ha,T.D.X., 2009, Optimality conditions for several types of efficient solutions of set-valued optimization problems,in: P. Pardolos, Th.M. Rassis, A.A. Khan (Eds.), Nnlinear Analysis and Variational Problems, Springer, p.305-324(Chapter 21).

[16] Hachimi, M. and Aghezzaf, B., 2007, New Results on Second-Order Optimality Conditions in Vector Optimization Problems, J. Optim. Theory Appl.,135,117-133.

[17] Jahn,J., 1986, Mathematical vector optimization in partially ordered linear space, Peter Lang, Frankfurt.

[18] Jahn,J., Khan,A.A., and Zeillinger, P., 2005, Second Order Optimality Conditions in Set Optimization, J. Optim. Theory Apply., 125,2,331-347.

[19] Jahn, J. and Rauh, R.,1997, Contingent Epiderivatives and Set-Valued Optimization Mathematical Methods of Operations Research, 46,193-211.

[20] Jimenez, B. and Novo, V., 2003, Second Order Necessary Conditions in Set Constrained Differentiable Vector Optimization, Mathematical Methods of Operations Research, 58,299-317. 
[21] Jimenez, B. and Novo, V.,2004, Optimality Conditions in Differentiable Vector Optimization via Second-Order Tangent Sets, Appl. Math. Optim., 49,123-144.

[22] Kasımbeyli,R.,2009 Radial Epiderivatives and Set-Valued Optimization, Optimization,58,5,519-532.

[23] Kalashnikov, V., Jadamba, B. and Khan,A.A., 2006, First and Second- Order Optimality Condition in Set-Optimization, In Optimization with Multivalued Mappings, Edited by: Dempe, S and Kalashnikov, V. , Berlin, Heidelberg: Springer Verlag, 265276.

[24] Kasımbeyli,R. and İnceoğlu, G.,2010, Optimality Conditions viaGeneralized Radial Epiderivatives in Nonconvex Set-Valued Optimization, In: R. Kasımbeyli, C. Dinçer, S. Özpeynirci and L. Sakalauskas (Eds.) Selected papers. 24th Mini EURO Conference on Continuous Optimization and Information-Based Technologies in the Financial Sector (24th MEC EurOPT 2010), June 23-26, 2010, Izmir University of Economics, Izmir, Turkey, ISBN: 978-9955-28-597-7, Vilnius ’Technika”, p. 148-154.

[25] Khan, A.A. and Tammer, C., 2013, Second Order Optimality Conditions in Set-Valued Optimization via Asymptotic Derivatives, Optimization, 62,6,743-758.

[26] Luc, D.T., 1991, Theory of Vector Optimization, Springer, Berlin.

[27] Németh, A.B., 1986, A Nonconvex Vector Minimization Problem, Nonlinear Anal.Theory Meth. Appl., 10,669-678.

[28] Penot, J.P., 1999,Second-Order Conditions for Optimization Problems Constraints, SIAM Journal on Optimization, 37,303-318.

[29] Sonntag, Y. and Zalinescu, C., 2000, Comparision of Existence Result for Efficient Points, J. Optim. Theory Appl.,105,161-188.

[30] Taa, A., 1998, Set-Valued Derivatives of Multifunctions and Optimality conditions, Numerical Functional Analysis Optimization, 19,121-140.

[31] Ward, D., 1993, Calculus for Parabolic Second-Order Derivatives. Set Valued Analysis, 1,213-246. 